\title{
Preliminary Study on Application Scope of New Ore Pulverizing Technology
}

\author{
Zhang Geng- Hao ${ }^{1,2}$, Feng Chun ${ }^{1,2}$, Fan Yong-Bo ${ }^{1,2, a *}$, Li Shi-Hai ${ }^{1,2}$ \\ ${ }^{1}$ Institute of mechanics, Chinese Academy of Sciences, Beijing, 100190, China \\ ${ }^{2}$ University of Chinese Academy of Sciences, Beijing, 100049
}

\begin{abstract}
New ore pulverizing technology was proposed by the Institute of mechanics, Chinese Academy of Sciences. In order to study the applicability of the new ore pulverizing technology to different materials, several experiments were carried out. In this paper, the author used the new ore pulverizing technology to pulverize iron ore, kaolin, wood and PVC, and finds that the new ore pulverizing technology has good application for iron ore, kaolin wood but for PVC. The experimental results show the application scope of the new ore pulverizing technology.
\end{abstract}

\section{Introduction}

A large number of ores are mined every year in the world. But most of the mined ores need to be powdered [1]. Ore pulverization is of great significance: First, the specific surface area of ore increases, which can increase its physical property or chemical reaction rate; Second, some ore after crushing can be used as aggregate with different particle size in concrete; Third, ore pulverization can separate the useful components in the ore [2]. Now the ore pulverization technology is mainly mechanical method, including the primary breaking, medium breaking, fine breaking, grinding and so on [3-4]. The main equipment for the ore primary breaking is jaw crusher [5-6], the main equipment for the ore medium breaking is hydraulic cone crusher [7-8], and the main equipment for the grinding is ball mill [9-10], vertical grinding mill and Raymond mill [11-13]. The traditional crushing and pulverizing process of ore takes a long time. In addition, during the whole crushing and pulverizing process, the ore is mainly destroyed by compression, which is inconsistent with the mechanical characteristics of the ore whose compressive strength is much higher than tensile strength [14-15]. A large amount of energy is consumed. In addition to the conventional mechanical crushing of ore, there are many other ways to pulverize ore. In the 1960s, former Soviet Union scientist Elperin proposed impinging stream technology and researched it in depth [16]. The principle of impinging stream technology is to use opposing high-speed airflow to collide, resulting in high turbulence and great impact velocity to powder materials [17]. The impinging stream technology is widely used in the production of ultrafine powder and material drying. Another new technology is the use of high-pressure liquid carbon dioxide to produce particles. Carbon dioxide, as an important component of the atmospheric cycle, has a large number of applications in industrial production [18]. The liquid carbon dioxide blaster was first invented by Aiduokesi company in the 1960 s, and then widely used in coal mining and rock fracturing and other fields [19-20]. Thirty years ago, some scholars found that the high-pressure liquid carbon dioxide can also be used to produce ore particles [21].

At present, with the decrease of high-grade ore, the increase of low-grade ore, the demand for ore pulverization is increasing [22]. What's more, countries all over the world are more and more concerned about environmental protection. This means that the ore pulverization technology not only meets the needs of industry, but also be as energy-saving and environmental protection as possible. Therefore, the study of ore pulverization technology is gradually becoming a hot spot. The Institute of mechanics, Chinese Academy of Sciences proposed a new technology of ore pulverization [23]. The principle of the new pulverization technology is to inject high-pressure liquid carbon dioxide into the ore, and then the ore unloaded at high speed. The highpressure liquid carbon dioxide inside the ore expands rapidly, and the ore collapses from the inside to the outside. Fan Yong-bo et al have verified the feasibility of the new pulverization technology by experiments [24-25].

In this paper, in order to study the applicability of the new pulverizing technology to different materials, the author uses the new ore pulverizing technology to pulverize iron ore, kaolin wood and PVC. And finds that the new ore pulverizing technology has good applicability for materials with a large number of micro pores, but for materials without internal micropores.

\section{The new pulverizing technology}

In order to study the applicability of the new pulverizing technology to different materials, the new pulverizing technology was deeply studied. And the experimental

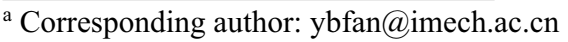


model of the new pulverizing technology was made in the laboratory for experimental research. Illustrate in Fig. 1-2

The experimental process of the new ore pulverizing technology is as follows: Firstly, the ore is placed in the high pressure chamber; Secondly, the high pressure liquid carbon dioxide is injected into the high-pressure chamber through the needle valve to create a highpressure environment for the ore; Thirdly, connect the wires and detonate the explosive (The main components of the nonel tube are potassium perchlorate, salicylic acid and ammonium oxalate.); Fourth, the rupture disk is broken instantly, and the high-pressure liquid carbon dioxide in the high-pressure chamber is rapidly vaporized and ejected, at the same time the ore is also sprayed into the atmosphere [24-25].

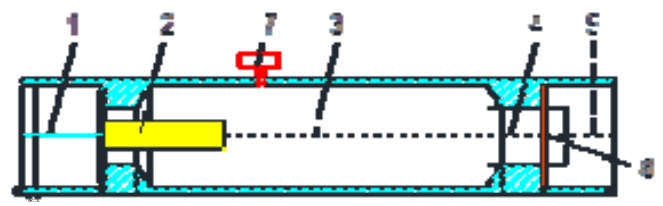

1- wire; 2- Nonel tube; 3- High pressure chamber; 4- Unloading port; 5-Spout; 6- Rupture disk; 7-Needle valve

Fig.1 Experimental apparatus model

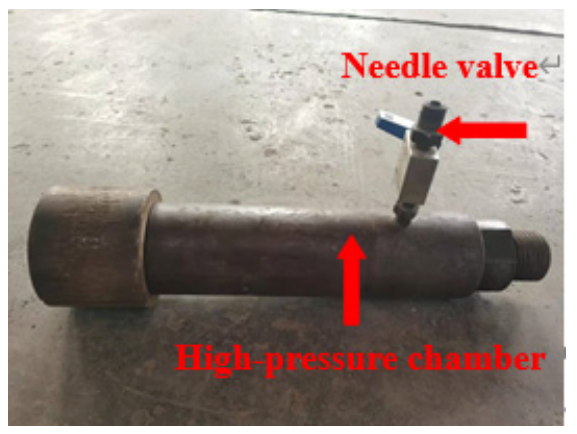

Fig.2 Experiment apparatus

During the above experiments, the ore was powdered. The speed of the whole ore pulverization process is very fast and completed in tens of milliseconds. In the process of ore pulverization, tensile failure occurs mainly, which saves energy a lot. Compared with the traditional technology, the new ore pulverizing technology has obvious advantages.

\section{Pulverizing ore with new ore pulverizing technology}

\subsection{Experiment of iron ore pulverization}

According to incomplete statistics, the annual output of iron ore in 2018 is 2.5 billion tons [26]. Crushing and pulverizing these iron ores is a huge project, which will cause a lot of energy consumption and environmental pollution. If the new pulverization technology can be applied to iron ore crushing and pulverization, the energy consumption and environmental pollution will be greatly reduced. Therefore, the experiment of using the new pulverizing technology to pulverize iron ore was carried out.

The experimental process is as follows: First, cooling the carbon dioxide to - 50C; Secondly, a nonel tube containing $70 \mathrm{~g}$ of explosives is installed inside the highpressure chamber; Third, put the iron ore into the highpressure chamber. The size of the ore is $2-3 \mathrm{~cm}$. Illustrate in Fig.3. Then, the carbon dioxide was pressurized to $10 \mathrm{MP}$ to be liquid and injected into the high-pressure chamber; Finally, connect the wire and detonate the explosive. After the experiment, open the experimental equipment, take out the iron ore powder.

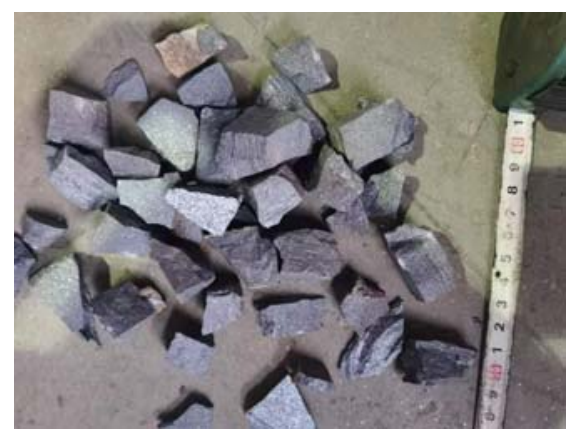

Fig.3 Characteristic size of iron ore

During the experiment, the iron ore was powdered. Illustrate in Fig.4. And the particle distribution curve was made. It can be seen from the particle distribution curve that iron ore is pulverized into extremely fine particles, of which more than $60 \%$ of particles are less than $0.5 \mathrm{~mm}$ in diameter. Illustrated in Fig.5. This means that new ore pulverization technology can pulverize iron ore.

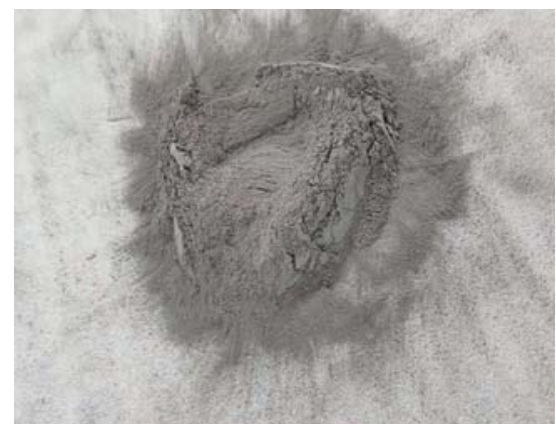

Fig.4 The iron ore powder

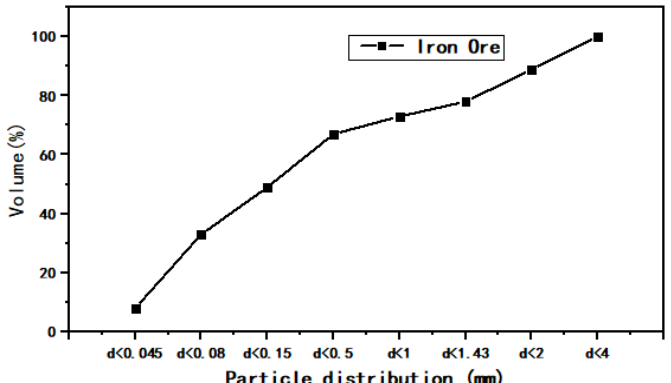

Fig.5 Particle size distribution of iron ore powder

\subsection{Experiment of kaolin pulverization}

According to statistics, the global production of kaolin 
has reached 43 million tons in 2019 [27]. The mined kaolin must be crushed and powdered. If the new pulverization technology can be applied to the crushing and pulverization of kaolin, it will be of great significance.

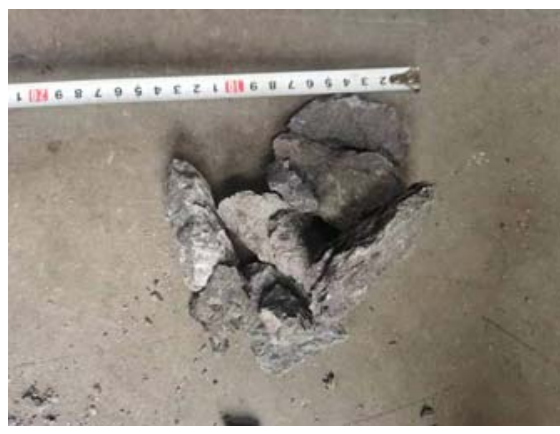

Fig.6 Characteristic size of kaolin

Therefore, we carried out the experiment of using new pulverizing technology to pulverize kaolin. The size of kaolin is $2-5 \mathrm{~cm}$. Illustrate in Fig.6. The experimental process of kaolin is similar to that of iron ore: The conditions are the same except that carbon dioxide is pressurized to $15 \mathrm{MPa}$. After the experiment, open the experimental equipment, take out the ore powder.

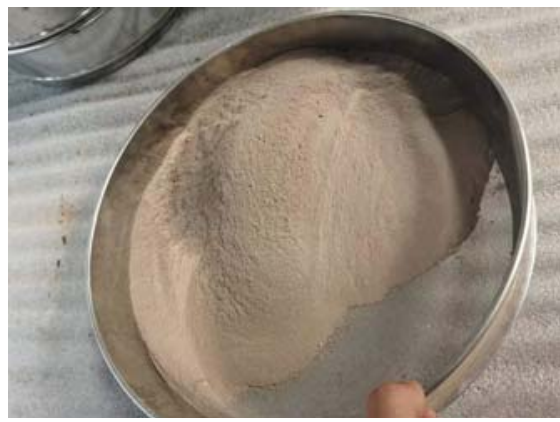

Fig.7 The kaolin powder

During the experiment, the iron ore was powdered. Illustrate in Fig.7. The particle distribution curve of ore powder is made. It can be seen from the particle distribution curve that kaolin is powdered into tiny particles. And about $70 \%$ of particles are less than $0.5 \mathrm{~mm}$ in diameter. This means that the new pulverization technology can be applied to the crushing and pulverization of kaolin after some improvement. Illustrate in Fig.8. This means that the new ore pulverization technology can pulverize kaolin.

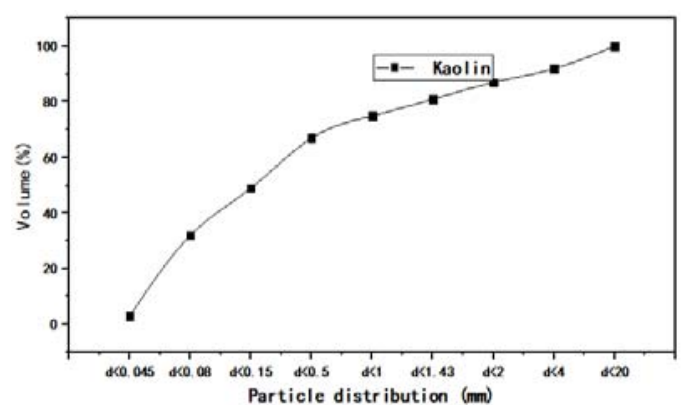

Fig.8 Particle size distribution of kaolin powder

\section{Application of new pulverizing technology to pulverize other materials}

\subsection{Experiment of wood pulverization}

Medicinal powder and wood powder is widely used in industrial production and pharmaceutical manufacturing. Materials like wood and medicinal materials also have a lot of pores in them [28]. Liquid carbon dioxide can be injected into the wood. Therefore, we suspect that the new pulverizing technology may also be used to pulverize these materials. The experiment of using new pulverizing technology to pulverize wood was carried out in the laboratory. Illustrate in Fig.9.

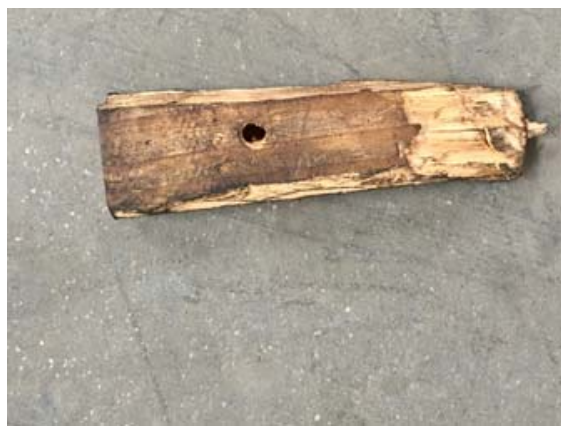

Fig.9 The wood for experiments

The experimental process is as follows: First, pre cool the carbon dioxide at - 50C; Secondly, a nonel tube containing $80 \mathrm{~g}$ of explosives is installed inside the highpressure chamber; Third, put the wood into the highpressure chamber. Then, the carbon dioxide was pressurized to 10MP to be liquid and injected into the high-pressure chamber; Finally, connect the wire and detonate the explosive. After the experiment is completed, open the high chamber, and take out the wood powder.

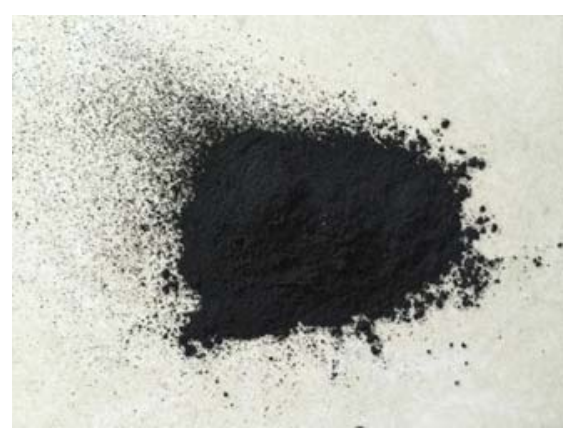

Fig.10 The wood powder

During the experiment, the wood was powdered. Illustrate in Fig.10. Some powdered wood was taken for laser particle analysis. The particle distribution curve of ore powder is made. It can be seen from the particle distribution curve that wood is powdered into micron sized particles. Among the fine particles, $30 \mu \mathrm{m}$ to $50 \mu \mathrm{m}$ accounted for the highest proportion. Illustrate in Fig. 11. This means that the new technology of ore pulverization can pulverize not only ore but also other materials. 


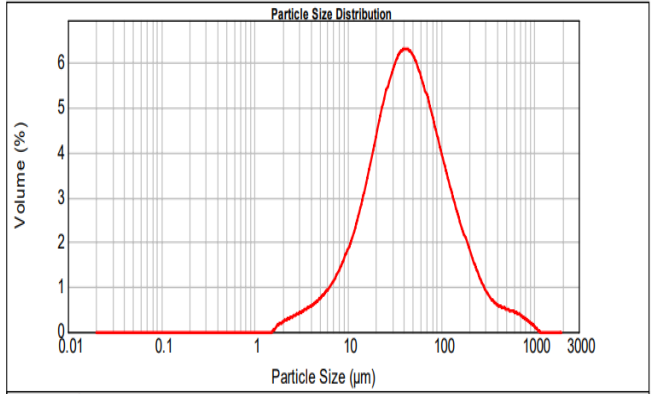

Fig.11 Particle size distribution of wood powder

\subsection{Experiment of PVC pulverization}

In order to study the applicability of new ore pulverization technology to non-porous materials. During the experiment of pulverizing kaolin, a $10 \mathrm{~cm}$ long PVC pipe was put in the high-pressure chamber. Illustrate in Fig. 12.

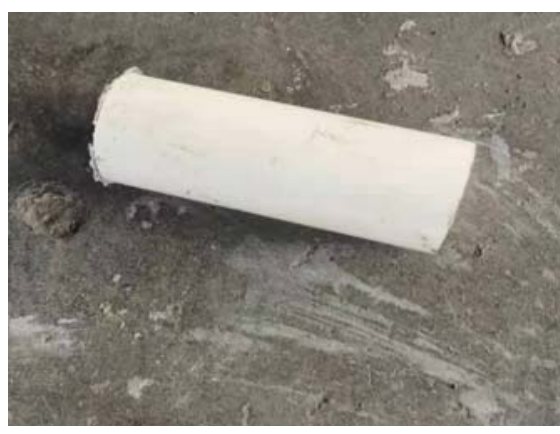

Fig.12 PVC tube

After the experiment, PVC was selected from kaolin powder. PVC is broken into small pieces, but no pulverization occurs. Illustrate in Fig.13.

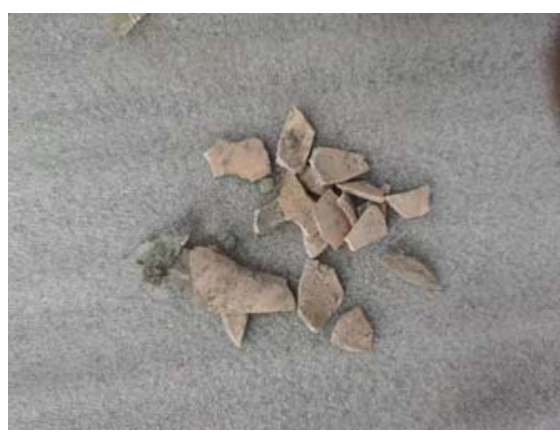

Fig.13 PVC fragment

The experimental results show that the effect is very limited for the use of new pulverizing technology to pulverize such dense materials as PVC. The main reason for this phenomenon is that PVC is a dense material and cannot be injected with high-pressure liquid carbon dioxide, so it can't be powdered.

\section{Conclusion}

Compared with the traditional ore pulverization technology, the new ore pulverization technology has the advantages of fast speed, less energy consumption, energy saving and less environmental pollution.

After a series of experiments, it can be found that the new pulverizing technology can pulverize materials with micro pores such as iron ore, kaolin and wood, but it can't be used for materials without pores like PVC. The main reason for this phenomenon is that there are micro pores in the material. The high-pressure liquid carbon dioxide can be injected into the material. However, materials without micropores or with few pores can't be injected with high-pressure liquid carbon dioxide and can't be powdered.

\section{References}

1. H.G. Lu, Introduction to powder technology [M]. Tongji University Press (1998)

2. M. Zhao, Y.P. Lu, Y.M. Pan, MAM, 10 (2): 36-41 (2001)

3. H.J. Li, X.D. Li, CME, 05: 10-13 (2006)

4. V.I. Molchanov, O. G.Selezneva, MAMO, 6 :587598 (1980)

5. M. Fladvad, T. Onnela, ME,151 (2020)

6. X. Wang, X.O. Xia, X.J. Luo, B. Chen, F.M. Liu, CM, 27 (S2): 227-230 (2018)

7. L.F. Ma, F.B.Wu, W.Q. Pan, G.H. Zhao, J. Zhang, HM, 05: 9-13 (2020)

8. V.V. Gabov, V.S. Romanova, EES, 87 :2 (2017)

9. P. Zhu, VE, 34(02):42-43 (2015)

10. M. Karuppannan, Y. Kim, D. Lee, JPE, 50:11191128 (2020)

11. A.L.R. Oliveira, V.A. Rodriguez, R.M. de Carvalho, et al. JE, 156 (2020)

12. S. Ehara, SE, 36(3):138-142 (1989)

13. W. Wang, SDIT, 11 (2018)

14. L. Sun, ASTI, 14: 48-49 (2012)

15. A. Teymen, E. CemalMengüç, IJMST, 30 :785-797 (2020)

16. IT. Elperin, JEP, 56(6):62-68 (1961)

17. Z.G. Sun, W.F. Li, H.F. Liu, Z.H. Yu, CRET, 25 (02): 97-103 (2009)

18. B. Dincer, C. Zamfirescu, Sustainable Energy Systems and Applications [M], 1-816 (2011)

19. Z.X. Guo, B, 72-74 (1994)

20. P. Shao, Y. Xu, Y.S, EM, 26 (5) : 6-8 (1997)

21. W.M. Dean, L.F. John, C.P. Ind. Eng. Chem. Res, 26: 2298-2306 (1987)

22. J.F. Yang, T. Ma, Y. Wang, C.G, CMM, 29 (10): 1-7 $+1(2020)$

23. Y.B. Fan, S.H. Li, W.J. Duan, J.Y. Qiao, C. Feng, L. Zhang, D.S. Zhang, A device and method for preparing iron ore powder by liquid $\mathrm{CO}_{2}[\mathrm{P}]$. Beijing: CN106733054b, February (2019)

24. Y.B. Fan, J.Y. Qiao, S.H. Li, C. Feng,

JACS, 55(2),595-600 (2019) 
25. Y.B. Fan, S.H. Li, W.J. Duan, J.Y. Qiao, P T,327,449-455 (2018)

26. Q. Wang, RIE, 33 (03): 59-68 (2020)

27. U.S. Geological Survey. Mineral Commodity Summaries 2019 (2020)

28. S. He, Y.H. Chen, Z. X. Wu, JFE, 12: 255 (2014) 